\title{
Detection of Methicillin Resistance in Staphylococcus aureus Isolated from Pediatric Patients: Is the Cefoxitin Disk Diffusion Test Accurate Enough?
}

\author{
Mimica M.J. ${ }^{1}$, Berezin E.N. ${ }^{1}$, Carvalho R.L.B. ${ }^{2}$, Mimica I.M. ${ }^{2}$, Mimica L.M.J. ${ }^{2}$, Sáfadi M.A.P. ${ }^{1}$, \\ Schneider E. ${ }^{2}$ and Caiaffa-Filho H.H. ${ }^{2,3}$ \\ ${ }^{I}$ Division of Pediatric Infectious Diseases, Department of Pediatrics; ${ }^{2}$ Laboratory of Microbiology, Department of Pathology \\ Santa Casa University Hospital; ${ }^{3}$ Laboratory of Medical Investigation - LIM 03, Hospital das Clinicas-FMUSP; São Paulo, SP, Brazil
}

\begin{abstract}
We evaluated the performance of several methods for the detection of methicillin resistance in Staphylococcus aureus using 101 clinical $S$. aureus isolates from pediatric patients in a tertiary hospital in Brazil; 50 isolates were $m e c A$-positive and 51 were $m e c A$-negative. The Etest and oxacillin agar screening plates were $100 \%$ sensitive and specific for $m e c A$ presence. Oxacillin and cefoxitin disks gave sensitivities of 96 and $92 \%$, respectively, and $98 \%$ specificity. Alterations of CLSI cefoxitin breakpoints increased sensitivity to $98 \%$, without decreasing specificity. Our results highlight the importance of a continuing evaluation of the recommended microbiological methods by different laboratories and in different settings. If necessary, laboratories should use a second test before reporting a strain as susceptible, especially when testing strains isolated from invasive or serious infections. With the new (2007) CLSI breakpoints, the cefoxitin-disk test appears to be a good option for the detection of methicillin resistance in $S$. aureus.
\end{abstract}

Key-Words: MRSA, disc diffusion, cefoxitin, mecA.

Methicillin-resistant Staphylococcus aureus (MRSA) has become a major epidemiological and clinical problem over the last decades. These strains have spread worldwide, causing nosocomial and, more recently, community-based infections [1]. This has led to the overuse of glycopeptides, and to the emergence of vancomycin-resistant $S$. aureus [2]. In this setting, rapid and accurate detection of methicillin resistance would help ensure correct use of antibiotics and appropriate epidemiological control of MRSA. Methicillin resistance in $S$. aureus is primarily mediated by overproduction of PBP2a protein, an altered penicillin-binding protein with lower affinity for beta-lactam antibiotics than PBP2, the main physiological methicillin target. PBP2a is encoded by the mecA gene, a component of a larger DNA fragment designated the mec region. Phenotypic expression of resistance may vary depending on culture conditions, such as temperature or osmolarity of the medium, despite genetic homogeneity [3].

This heterogeneous resistance phenotype may complicate the detection of MRSA by conventional susceptibility methods. Oxacillin-disk diffusion has been the traditional method for methicillin-resistance routine screening; but recently good accuracy of the cefoxitin disk for predicting methicillin resistance has been reported [4-11], and CLSI (Clinical and Laboratory Standards Institute) has recommended that cefoxitin should be preferred over oxacillin for the detection of $m e c A$-mediated resistance [4]. However,

Received on 5 March 2007; revised 8 July 2007.

Address for correspondence: Dr. Marcelo Jenné Mimica. Rua Cesário Motta Júnior, 61, décimo andar, Santa Cecília. Zip code: 01221-020. São Paulo, SP, Brazil. E-mail: mjmimica@ hotmail.com. Phone: 5511 81752258. Financial support: M.J. Mimica received financial support from CAPES (Coordenação de Aperfeiçoamento de Pessoal de Nível Superior).

The Brazilian Journal of Infectious Diseases

2007;11(4):415-417. (C) 2007 by The Brazilian Journal of Infectious Diseases and Contexto Publishing. All rights reserved. the choice of the best phenotypic method for detecting methicillin resistance in S. aureus remains controversial. Good accuracy of other methods, such as Etest and oxacillin-agar screening plate, has also been demonstrated [12-15]. Detection of the gene is considered the reference method [3], but this is not feasible in most laboratories throughout the world.

Our main objective was compare oxacillin and cefoxitindisk tests, Etest and oxacillin-agar screening plates for detection of methicillin resistance in S. aureus, using PCR for $m e c A$ as the "gold standard" comparison assay.

\section{Materials and Methods \\ $\underline{\text { Strains }}$}

We studied 101 pediatric clinical isolates of S. aureus (isolated from different anatomical sites of different pediatric patients) that were collected from April 2004 to June 2005 and identified by biochemical procedures. Staphylococcus aureus ATCC 25923, ATCC 29213 and ATCC 33591 were used as quality-control strains.

\section{Detection of the mecA Gene}

A single bacterial colony was obtained from a fresh subculture and was resuspended in $25 \mu \mathrm{L}$ of sterile water. The suspension was boiled at $95^{\circ} \mathrm{C}$ for DNA extraction. One microliter of the DNA samples was added to $19 \mu \mathrm{L}$ of PCR mixture, consisting of $1 \mathrm{U}$ Taq polymerase, $1 \mathrm{X}$ polymerase buffer [ $50 \mathrm{mM} \mathrm{KCl}, 20 \mathrm{mM}$ Tris- $\mathrm{HCl}$ ( $\mathrm{pH} 8.4$ ), $1.5 \mathrm{mM} \mathrm{MgCl}_{2}$ ], $200 \mu \mathrm{M}$ dNTPs mixture and $0.5 \mu \mathrm{M}$ of each primer. Amplification was performed using a Perkin Elmer 2400 thermocycler (Applied Biosystems, California, USA). After an initial denaturation step (three minutes at $94^{\circ} \mathrm{C}$ ), 30 cycles of amplification were performed: denaturation at $94^{\circ} \mathrm{C}$ for one minute, annealing at $56^{\circ} \mathrm{C}$ for one minute and DNA extension at $72^{\circ} \mathrm{C}$ for one minute. The reaction was finished with a final extension step at $72^{\circ} \mathrm{C}$ for seven 
minutes. The set of primers used (M1, 5'TGGCTATCGTGTCACAATCG-3' and M2, 5' CTGGAACTTGTT-GAGCAGAG-3') was described by Vannuffel [16]. The amplified product was a 310-bp DNA fragment that was detected by $1.5 \%$ agarose gel electrophoresis, with ethidium bromide staining visualized under UV light.

\section{Susceptibility Tests}

The isolates were tested with oxacillin $(1 \mu \mathrm{g})$ and cefoxitin (30 $\mu \mathrm{g})$ disks, using Mueller-Hinton agar plates inoculated with a suspension (equivalent to a $0.5 \mathrm{McFarland}$ standard) of the $S$. aureus clinical isolates. The plates were incubated at $35^{\circ} \mathrm{C}$ for 24 hours and inhibition zones were measured. The oxacillin MICs (minimum inhibitory concentration) were determined by Etest (AB Biodisk, Solna, Sweden) using Mueller-Hinton agar plates supplemented with $2 \% \mathrm{NaCl}$. The CLSI 2005 criteria [4] were used for interpretation (Table 1). Isolates were also tested with oxacillin agar screening, which was performed by inoculating a direct colony suspension (0.5 McFarland standard) with a swab, spotting an area 10 to $15 \mathrm{~mm}$ in diameter, on Mueller-Hinton agar supplemented with $4 \% \mathrm{NaCl}$ and oxacillin at $6 \mathrm{mg} / \mathrm{L}$. After incubation for 24 hours, any growth was interpreted as a positive result for MRSA.

\section{Results and Discussion}

Among the 101 strains included in our study, 50 were mecApositive and 51 were mecA-negative. The results of the phenotypic tests are shown in Table 2. Etests and oxacilin plates were the most accurate methods. Both were $100 \%$ sensitive and specific.
In other studies that used the presence of mecA as the gold standard, the accuracy of these techniques was also very good [12-15].

The oxacillin and the cefoxitin disk tests showed sensitivities of $96 \%$ and $92 \%$, respectively, and $98 \%$ specificity. Six strains had discrepant results between at least one of the disks and the mecA gene (Table 3). The accuracy of the cefoxitin disk test in our study was different from that previously reported. However, among the four mecA-positive strains giving false-negatives by the cefoxitin test, three had an inhibition zone of $20 \mathrm{~mm}$, and none of the mecA-negative strains had such a large zone diameter. Thus, if there were changes in the cefoxitin breakpoints, the sensitivity of the method could be increased, perhaps without any decrease of specificity. Other authors have also suggested different breakpoints in the interpretative zone diameters of cefoxitin for better detection of methicillin resistance in $S$. aureus $[7,10]$.

We evaluated our strains in 2005, using the former CLSI cefoxitin breakpoints (R: $\leq 19 \mathrm{~mm} ; \mathrm{S}: \geq 20 \mathrm{~mm}$ ). But, in 2007 the recommended CLSI breakpoints were changed [17], in order to increase the accuracy of the method. With the new breakpoints (R: $\leq 21 \mathrm{~mm} ; \mathrm{S}: \geq 22 \mathrm{~mm}$ ), the three strains that had a $20 \mathrm{~mm}$ zone would have been correctly diagnosed, increasing the sensitivity to $98 \%$, again without any decrease in specificity, since we did not find any $m e c A$-negative strains with inhibition zones smaller than $22 \mathrm{~mm}$.

Although the number of isolates tested in our study was low and there was a chance of clonality, our results highlight the importance of a continuing evaluation of recommended microbiological methods by different laboratories and in different settings. If necessary and if the evidence supports it,

Table 1. CLSI 2005 Interpretative criteria for methicillin resistance in Staphylococcus aureus isolated from pediatric patients [4].

\begin{tabular}{llll}
\hline & Inhibition zone $(\mathbf{m m})$ & & \\
& $\mathrm{R}$ & $\mathrm{I}$ & $\mathrm{S}$ \\
Oxacillin disk & $\leq 10$ & $11-12$ & $\geq 13$ \\
Cefoxitin disk & $\leq 19$ & - & $\geq 20$ \\
\cline { 2 - 3 } & Minimum inhibitory concentration $(\boldsymbol{\mu g} / \mathbf{m L})$ & $\mathrm{S}$ \\
Oxacillin & $\mathrm{R}$ & - & $\leq 2$ \\
\hline
\end{tabular}

R: resistant; S: susceptible; I: intermediate.

Table 2. Results of the phenotypic tests of Staphylococcus aureus isolated from pediatric patients and correlation with mecA.

\begin{tabular}{|c|c|c|c|c|c|c|c|c|}
\hline & \multicolumn{2}{|c|}{ Etest } & \multicolumn{2}{|c|}{$\begin{array}{c}\text { Agar } \\
\text { screening }\end{array}$} & \multicolumn{2}{|c|}{$\begin{array}{c}\text { Oxacillin } \\
\text { disk }\end{array}$} & \multicolumn{2}{|c|}{$\begin{array}{c}\text { Cefoxitin } \\
\text { disk }\end{array}$} \\
\hline & $\mathbf{R}$ & $\mathbf{S}$ & $\mathbf{R}$ & $\mathbf{S}$ & Rou I & $\mathbf{S}$ & $\mathbf{R}$ & $\mathbf{S}$ \\
\hline mecA+ & $50 / 50$ & $0 / 50$ & $50 / 50$ & $0 / 50$ & $48 / 50$ & $2 / 50$ & $46 / 50$ & $4 / 50$ \\
\hline mecA- & $0 / 51$ & $51 / 51$ & $0 / 51$ & $51 / 51$ & $1 / 51$ & $50 / 51$ & $1 / 51$ & $50 / 51$ \\
\hline
\end{tabular}

R: resistant; S: susceptible. 
Table 3. Isolates with discrepant results between at least one of the disks and the mecA gene in Staphylococcus aureus isolated from pediatric patients

\begin{tabular}{|c|c|c|c|c|c|c|c|}
\hline Isolate & mecA & $\begin{array}{l}\text { Oxacillin } \\
\text { (mm) }\end{array}$ & & $\begin{array}{c}\text { Cefoxitin } \\
\text { (mm) }\end{array}$ & & $\begin{array}{c}\text { Etest } \\
(\mu \mathrm{g} / \mathrm{mL})\end{array}$ & $\begin{array}{c}\text { Agar } \\
\text { screening }\end{array}$ \\
\hline 1 & - & 12 & I & 16 & $\mathrm{R}$ & 0.5 & $\mathrm{~S}$ \\
\hline 2 & + & 0 & $\mathrm{R}$ & 20 & $\mathrm{~S}$ & $>256$ & $\mathrm{R}$ \\
\hline 3 & + & 14 & $\mathrm{~S}$ & 18 & $\mathrm{R}$ & $>256$ & $\mathrm{R}$ \\
\hline 4 & + & 16 & $\mathrm{~S}$ & 20 & $\mathrm{~S}$ & $>256$ & $\mathrm{R}$ \\
\hline 5 & + & 0 & $\mathrm{R}$ & 20 & $\mathrm{~S}$ & $>256$ & $\mathrm{R}$ \\
\hline 6 & + & 0 & $\mathrm{R}$ & 22 & $\mathrm{~S}$ & $>256$ & $\mathrm{R}$ \\
\hline
\end{tabular}

R: resistant; S: susceptible.

the method should be withdrawn from the recommendations or its breakpoints changed. In addition, laboratories could use a second test before reporting a strain as susceptible, especially when testing strains from invasive or serious infections.

\section{Conclusion}

In our study the oxacillin agar screening plate appeared to be a good option for the detection of methicillin resistance in $S$. aureus, due to its great accuracy and low cost. With the new (2007) CLSI breakpoints, the cefoxitin-disk test may also be a reasonable alternative.

\section{References}

1. Chambers H.F. The changing epidemiology of Staphylococcus aureus? Emerg Infect Dis 2001;7:178-82.

2. Centers for Disease Control and Prevention (CDC), Staphylococcus aureus resistant to vancomycin - United States, 2002. Morb Mortal Wkly Rep 2002;51:565-7.

3. Chambers H.F. Methicillin resistance in staphylococci: molecular and biochemical basis and clinical implications. Clin Microbiol Rev 1997; 10:781-91.

4. Clinical and Laboratory Standards Institute. Performance standards for antimicrobial susceptibility testing, fifteenth informational supplement, document M100-S15. CLSI, Wayne, Pa, USA, 2005.

5. Pottumarthy S., Fritsche T.R., Jones R.N. Evaluation of alternative disk diffusion methods for detecting $m e c A$-mediated oxacillin resistance in an international collection of staphylococci: Validation report from the SENTRY Antimicrobial Surveillance Program. Diagn Microbiol Infect Dis 2005;51:57-62.

6. Velasco D., Tomas M.M.T., Cartelle M., et al. Evaluation of different methods for detecting methicillin (oxacillin) resistance in Staphylococcus aureus. J Antimicrob Chemother 2005; $55: 379-82$.

7. Skov R., Smyth R., Clausen M., et al. Evaluation of a cefoxitin $30 \mu \mathrm{g}$ disc on Iso-sensitest agar for detection of methicillin-resistant Staphylococcus aureus. J Antimicrob Chemother 2003;52:204-7.
8. Fernandes C.J., Fernandes L.A., Collignon P. Cefoxitin resistance as a surrogate marker for the detection of methicillin-resistant Staphylococcus aureus. J Antimicrob Chemother 2005;55:50610.

9. Cauwelier B., Gordts B., Descheemaecker P., et al. Evaluation of a disk diffusion method with cefoxitin (30 microg) for detection of methicillin-resistant Staphylococcus aureus. Eur J Clin Microbiol Infect Dis 2004;23:389-92.

10. Felten A., Grandry B., Lagrange P.H., et al. Evaluation of three techniques for detection of low-level methicillin-resistant $S$. aureus (MRSA): a disk diffusion method with cefoxitin and moxalactam, the Vitek 2 system, and the MRSA-screen latex agglutination test. J Clin Microbiol 2002;40:2766-71.

11. Swenson J.M., Tenover F.C. Results of disk diffusion testing with cefoxitin correlate with presence of mecA in Staphylococcus spp. J Clin Microbiol 2005;43:3818-23.

12. Kohner P., Uhl J., Kolbert C., et al. Comparison of susceptibility testing methods with mecA gene analysis for determining oxacillin (methicillin) resistance in clinical isolates of Staphylococcus aureus and coagulase-negative Staphylococcus spp. J Clin Microbiol 1999;37:2952-61.

13. Sakoulas G., Gold H.S., Venkataraman L., et al. Methicillin-resistant Staphylococcus aureus: comparison of susceptibility testing methods and analysis of $m e c A$-positive susceptible strains. J Clin Microbiol 2001;39:3946-51.

14. Sharp S.E., Warren J.A., Thomson R.B. Cefoxitin disk diffusion screen for confirmation of oxacillin-resistant Staphylococcus aureus isolates and utility in the clinical laboratory. Diagn Microbiol Infect Dis 2005;51:69-71.

15. Weller T.M.A., Crook D.W., Crow M.R., et al. Methicillin susceptibility testing of staphylococci by Etest and comparison with agar dilution and $m e c A$ detection. J Antimicrob Chemother 1997;39:251-3.

16. Vannuffel P., Gigi J., Ezzedine H., et al. Specific detection of methicillin-resistant Staphylococcus species by multiplex PCR. J Clin Microbiol 1995;33:2864-7.

17. Clinical and Laboratory Standards Institute. Performance standards for antimicrobial susceptibility testing, seventeenth informational supplement, document M100-S17. CLSI, Wayne, Pa, USA, 2007. 